\title{
Fitness Assessment Feedback May Lower Intrinsic Motivation for Physical Activity among College Students
}

\author{
Laura Chandler $^{1}{ }^{(0}$, Jerry W. Lee ${ }^{2}$ (), Karen T. Lesniak ${ }^{3}$, and R. Patti Herring ${ }^{4}$ (i) \\ ${ }^{1}$ Dept. of Public Health, California State University Fullerton, Fullerton, California; USA \\ ${ }^{2}$ Dept. of Health Education, School of Public Health, Loma Linda University, Loma Linda, Ca \\ ${ }^{3}$ School of Behavioral Health, Loma Linda University, Loma Linda, Ca; (Retired) \\ ${ }^{4}$ Dept. of Health Education, School of Nursing, Loma Linda University, Loma Linda, Ca
}

\begin{abstract}
Background and Purpose: Fitness assessments are commonly used as a motivational tool in exercise classes and fitness training. However, there is little research on their actual effect. This study explored how the feedback from a fitness assessment may affect intrinsic motivation for physical activity in college students. Methods: The study utilized a quasi-experimental design where 430 college students were assigned to either an intervention or one of two control groups. The fitness assessment was only distributed to the subjects in the intervention group. Students were surveyed at four-time points to examine subjects' competence, autonomy and intrinsic motivation. A repeated measures general linear model measured differences between those who did and did not receive the fitness assessment. Results: Intrinsic motivation $(F(6,848)=2.33 p=.031)$ and competence $(F(6,848)=3.81, p=.001)$ diminished significantly in the group receiving fitness assessment feedback as compared to either control group. Additionally, for those in the intervention group that perceived their feedback as negative there was a significant decrease in competence $(\mathrm{F}(1,155)=15.59, \mathrm{p}<.001)$, intrinsic motivation $(F(1,155)=6.41, p=.012)$, and physical activity $(F(1,155)=7.46, p=.007)$. Conclusion: Fitness assessment feedback may hinder intrinsic motivation toward physical activity at least among those dissatisfied with the feedback.
\end{abstract}

(C) 2021 and CC-BY 4.0 licensed by the authors.

Keywords: physical activity, fitness assessment, motivation, self determination

\section{Introduction}

More Americans are classified as overweight and obese than just 30 years ago. In fact, there has been a gradual increase in body mass index (BMI) since the 1980's (Centers for Disease Control and Prevention, 2015). Much attention has gone into deciphering this trend. Researchers have attributed it to, among other factors, an increase in sedentary behavior. Further merit is given as Healthy People 2030 includes 23 core objectives dedicated to addressing the increase of physical activity among various groups and across the lifespan (US Department of Health and Human Services, 2020).
The overall decline in physical activity among the U.S. population is not only alarming in relation to people being overweight and obese but also as it relates to premature death and disability from various chronic diseases including coronary heart disease, diabetes, and certain cancers. In addition, medical costs related to physical inactivity are staggering. One analysis found that the percentage of aggregate health care expenditures associated with inadequate levels of physical activity (i.e., inactive and insufficiently active) was $12.5 \%$ and remained significant at $11.1 \%$ after adjusting for BMI. That equates to an estimated $\$ 131$ billion before adjusting for BMI 
and $\$ 117$ billion after adjusting for BMI of health care expenditures per year associated with inadequate levels of physical activity (Carlsona, et al., 2015).

Youth, in particular, have experienced considerable decline in physical activity as schools eliminate non-essential course work to reduce their budgets even though national guidelines recommend 60 minutes of daily physical activity for children (US Department of Health and Human Services, 2018). In one study, researchers examined data from the Youth Risk Behavior Survey (YRBS) from 1991 to 2001 and found a decline in the percentage of students, from $41.6 \%$ to $32.2 \%$, who attended daily physical education classes (Brownson, et al., 2005). In 2017, data from YRBS showed a continued decline with only $29 \%$ of youth in the U.S. participated in daily physical education classes (CDC, 2017). Unfortunately, inactivity does not improve as youth age. The national guidelines from the US Department of Health and Human Services, recommend a minimum of 20 minutes of moderate to vigorous physical activity daily for adults, however data from the 2019, National College Health Assessment indicates only $56 \%$ of college students reported participating in moderate exercise for at least 30 mins 1-4 days per week, and only $26 \%$ in vigorous exercise for at least 20 minutes 3-7 days per week. Twenty-two percent of college students reported zero days per week of participation in at least 30 minutes of moderate exercise (American College Health Association, 2019). In addition, research shows that nearly half of all students report a decrease in physical activity following graduation from college (Calfas, et al., 1994).

Since their early use, fitness assessments have become a standard of physical education classes from elementary school through high school. The American College of Sports Medicine defines a physical fitness assessment as an appraisal that includes measures of body composition, cardiorespiratory endurance, muscular fitness, and musculoskeletal flexibility (Wilder, et. al,
2006). The California Department of Education (n.d.) requires all students to participate in fitness assessments in grades 5, 7, and 9. The U.S. Department of Education as reported by Shape of the Nation Report (2014) indicates that nearly all states mandate the use of fitness assessments by all students annually or biannually. It is surprising that with much use there has been very little research dedicated to fitness assessments in general or the connection between these assessments and motivation toward physical activity. In contrast, over the last few decades research has been strong in the area of motivation for physical activity and intrinsic motivation.

Deci and Ryan, (1985) have been at the forefront of this motivation research, as the creators of the self-determination theory (SDT) and its offspring cognitive evaluation theory (CET). The main construct of both SDT and CET is intrinsic motivation, which is described by Deci and Ryan (2000) as "the inherent tendency to seek out novelty and challenges, to extend and exercise one's capacities to explore and learn" (p.70). It is important to note that these theories do not focus on factors that cause intrinsic motivation but rather conditions that enhance versus diminish it. CET is based on two basic needs or constructs: competence and autonomy and their relation to intrinsic motivation. Competence meaning one's sense that they are able to do something successfully or efficiently and autonomy meaning one's sense that their behavior is voluntary or self-directed. For example, an event such as receiving positive feedback that creates a sense of competence in an individual during an action can enhance their intrinsic motivation for that action. Wiersma and Sherman (2008) found that competence can come through one's own assessment of their performance or through the feedback provided by others, such as teachers, coaches or peers. In addition, it is important to note that negative performance feedback as well as threats, imposed goals, directives and deadlines can diminish intrinsic motivation (Deci, 1975). 
For intrinsic motivation to be enhanced these feelings of competence must be accompanied by a sense of autonomy - a feeling that the action is self-directed and not controlled by others (Ryan $\&$ Deci, 2000). In an article reviewing SDT and CET in a number of domains including sports, education, and health care, Frederick-Recascin, (2002) found that across studies in all these areas, results clearly indicate that the degree of autonomy does influence affect and behavior.

Based on the above findings, it is logical that receipt of perceived positive feedback from a fitness assessment can create a sense of competence in individuals. In addition, it seems that with a positive perception there could also be a sense of autonomy in that the actions required to be at this assessment level were selfdetermined. Thus, intrinsic motivation would be enhanced. The reverse would likely occur if the fitness assessment feedback were perceived as negative (Figure 1). This is illustrated in a study by Whitehead and Corbin (1991) where individuals given bogus (manufactured by researchers) positive fitness feedback experienced enhanced intrinsic motivation toward physical activity and whereby bogus negative fitness feedback diminished their intrinsic motivation toward physical activity.

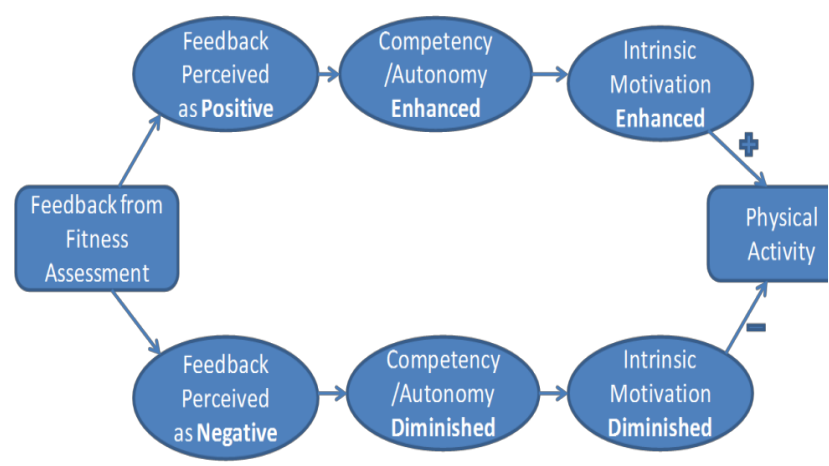

Figure 1: Self-determination theory constructs in relation to fitness assessment feedback and physical activity.

To date, there is no published research on the role of legitimate fitness assessment feedback and its influence on motivation toward physical activity.
However, a majority of community and university fitness facilities/labs provide individuals with some form of fitness assessment either on a voluntary basis or as a requirement for a specific course. In this study, the primary purpose was to explore the impact of feedback from a fitness assessment with regard to one's intrinsic motivation toward physical activity and physical activity levels. Further, this exploration was conducted based on the cognitive evaluation theory (CET) constructs of competence, autonomy, and intrinsic motivation.

\section{Methods}

\section{Study Design and Procedures}

This study utilized a quasi-experimental design as groups/classes, rather than participants, were randomly assigned. Eight health science classes (HESC) were randomly assigned to either a control group $(n=150)$ or an intervention group $(n=157)$ with four classes assigned to each group. The students in the HESC intervention group participated in a fitness assessment as part of the standard health course curriculum. The students in the HESC control group did not participate in the fitness assessment; however, they were given a voucher to participate in the assessment the following semester if they wished. Because students who chose to enroll in the health class may be more health conscious or physically active, three sections of an introductory sociology class (SOCI) served as an additional control group (SOCI control) $(n=123)$. With this group, we were also able to control for any change in participants that may have occurred simply from exposure to the health course curriculum.

Surveys were administered to students in the 11 classes at four time points during the 16-week semester. All variables (competence, autonomy, intrinsic motivation \& physical activity) were measured each time among all groups (Figure 2). Data collection followed this schedule:

Time 1 - week 1-2 of the semester, baseline; 
Time 2 - week 6-8, after fitness assessment (intervention) had been performed;

Time 3 - week 9-12, immediately after students received and reviewed their fitness assessment feedback - perception variable added;

Time 4 - week 15-16, end of the semester.

It is important to note that Time 1 is valuable as a collection of baseline data, particularly for the intervention group, as simply the experience of participating in the fitness assessment could have had an influence on the students' competence, autonomy or intrinsic motivation. The control groups followed the same data collection schedule even though they did not participate in the fitness assessment. Student anonymity and confidentiality of their data were preserved by use of a code containing the birthday, gender, and section of the course to which the student was enrolled. Students who participated in three of the four survey time points received a $\$ 5$ gift certificate to the university food court.

\begin{tabular}{|l|c|c|c|c|}
\hline & $\begin{array}{c}\text { Time 1 (week 1-2 of } \\
\text { the semester) }\end{array}$ & $\begin{array}{c}\text { Time 2 (week 6-8 of } \\
\text { the semester) }\end{array}$ & $\begin{array}{c}\text { Time 3 (week 9-12 } \\
\text { of the semester) }\end{array}$ & $\begin{array}{c}\text { Time 4 (week 15-16 } \\
\text { of the semester) }\end{array}$ \\
\hline $\begin{array}{l}\text { Intervention } \\
\text { Group }\end{array}$ & 0 & 0 & $\begin{array}{c}\mathrm{X}, 0 \\
\text { (perception variable } \\
\text { added to survey) }\end{array}$ & 0 \\
\hline HESC Control & 0 & 0 & 0 & 0 \\
\hline SOCI Control & 0 & 0 & 0 & 0 \\
\hline
\end{tabular}

$\mathrm{O}=$ Survey Administered; $\mathrm{X}=$ Fitness Assessment Results distributed to Students

Figure 2: Study design and data collection schedule

\section{Variables and Survey Instrument}

The fitness assessment (independent variable) done in the intervention group was performed by four trained kinesiology graduate students in the physical performance laboratory. Participants were assessed in the following seven areas: aerobic fitness, body composition, flexibility, muscular strength, pulmonary function, resting blood pressure, and total coronary heart disease (CHD) risk score (as determined by health behavior and family history questions). The feedback was given via a computer-generated document, from a programmed Microsoft Excel spreadsheet which was developed by the university exercise physiology lab director. The first page of the report provides the students' scores on each of seven areas. The second page provides these scores but presents them in a bar graph where students are compared to other like college students (male or female). The scale is a percentile scale for each fitness area and also includes the headings of "below average", "average" and "above average". The final page is a general description of each of the seven areas of fitness assessed and is not personalized.

Competence, autonomy and intrinsic motivation (dependent variables) were measured in the survey by utilizing three specific subscales from the Intrinsic Motivation Inventory. The Intrinsic Motivation Inventory has been used to measure subjective experience of physical activity in laboratory experiments (selfdeterminationtheory.org, 2010), and studies (e.g., McAuley, et al., 1989); Tsigilis \& Theodosiou, 2003) have found strong support for the validity and reliability of the Intrinsic Motivation Inventory. Each of the scales consisted of 5-7 items utilizing a 7-point scale ranging from 1 (not at all true) to 7 (very true). A sample item for each of the constructs follows: Competence, "I think I am pretty good at exercise/physical activity"; Autonomy, "I feel that it is my choice to engage in exercise/physical activity"; and Intrinsic Motivation, "I find exercise/physical activity very interesting." Based on Cronbach's alphas in the baseline data, the measures for Competence $(\alpha=.87)$, Autonomy 
$(\alpha=.74)$, and Intrinsic Motivation $(\alpha=.94)$ were reliable.

Physical activity levels (dependent variable) were assessed by six questions in the survey focusing on aerobic activity, muscle strengthening, and flexibility $(\alpha=.69)$ which was adapted from the National College Health Risk Behavior Survey (CDC, 1997). An example item reads, on how many of the past 7 days did you do exercises to strengthen or tone your muscles, such as pushups, sit-ups, or weight lifting? Adequate reliability was expected as this construct represents the variety of types of physical activity.

In addition to the above variables, a variable of perception was added for the intervention group (those that participated in the fitness assessment). This dependent variable measured the students' perception of their fitness assessment feedback. This was only done at Time 3, directly after the students received and reviewed their feedback from the fitness assessment. Four items $(\alpha=.92)$ created specifically for this study were used to measure these perceptions. Participants indicated their perception on a 7-point scale, ranging from 1 (discouraging) to 7 (encouraging), with various descriptive words related to each item (e.g., "Overall my fitness results are:"). A dichotomous variable was created where scores less than 4 were labeled as "negative perception" and scores of 4 or greater were labeled as "positive perception."

\section{Participants}

The participants in this study were undergraduate students from a large public university located in Southern California, with an enrollment of approximately 38,000 students. Recruitment of participants was conducted via outreach to Health Science professors of an introductory health course and Sociology professors of an introductory sociology course for the spring semester. The health course was selected, as a student fitness assessment is part of its standard curriculum. Eleven classes were involved in this study, eight health science classes (HESC) and three sociology classes (SOCI), each with 40 to 50 students. These courses are often taken as electives to meet general education requirements; therefore, the students represent a variety of academic majors.

A total of 430 participants were sampled, (156 males and 274 females), all of whom were aged from 18 to $29(\mathrm{M}=19.54, \mathrm{SD}=1.71)$. The sample consisted of 37\% Caucasian, 26\% Hispanic, 23\% Asian/Pacific Islander, 5\% Black/African American, less than 1\% American Indian and 8\% other. These ethnic breakdowns closely mimicked those of the university (32\% Caucasian, 28\% Hispanic, 22\% Asian/Pacific Islander, 4\% Black/African American, 1\% American Indian and 10\% other) (citation absent for blinding purposes).

The university's Institutional Review Board granted approval of the research study. Prior to data collection, the study was explained to the students in accordance with institutional review guidelines and students signed a form to verify their consent.

\section{Data Analyses}

Data were double entered, cleaned and analyzed with SPSS 16. Since this study was based on data collection from four different time points throughout the semester, students who only took one survey ( 82 cases) were omitted due to a lack of data for comparison. Of the 430 cases remaining, 230 students completed all four surveys. For the remaining 200 cases missing one or two surveys, missing value analysis was performed, and expectation maximization missing data imputation was done with SPSS 16. Paired $t$-tests assessed within-group differences for each group at the different time points. A repeated measures general linear model controlling for potential gender and racial/ethnic differences was then utilized to detect any differences between the intervention group and the two control groups. All data were examined for gender and ethnicity differences among the 
three groups, but no such differences were discovered.

\section{Results}

\section{Competence, Intrinsic Motivation and Autonomy}

This study compared the three groups (group 1, intervention; group 2, HESC control, and group 3, SOCI control) over each of the four time points, utilizing the general linear model for analysis and found interesting although not completely anticipated results.

There were no significant changes in any of the groups from Time 1 to Time 2; however, looking at other time points there were varying changes among the three groups (one intervention and two controls) in all three constructs; autonomy, competence, and intrinsic motivation (Figure 3). Compared to the two control groups, the intervention group had a significant decrease in competence from Time 2 to Time $3(F(6$, $848)=3.81, p=.001)$. Time 3 was the time point when students were allowed to review the feedback of their fitness assessments, and then were subsequently surveyed. Given the established relationship of competence and intrinsic motivation in the SDT and CET (as competence decreases, intrinsic motivation decreases) and based on the changes in competence among the groups over time, the observed similar changes in intrinsic motivation were expected. Both control groups remained fairly consistent in the intrinsic motivation scale; however in the intervention group, a decline in intrinsic motivation occurred relative to the two control groups once the intervention students received and reviewed their fitness feedback $(F(6,848)=2.33 p=.031)$. The intervention group experienced some movement back to baseline from Time 3 to Time 4 in both competence and intrinsic motivation; however neither the improvement in intrinsic motivation $(F(1,154)=0.02, \quad p=.888) \quad$ nor competence $(F(1,154)=0.002, \quad p=.961) \quad$ were significant. Autonomy decreased significantly for all three groups over the semester $(F(3,423)=4.25$, $p=.006)$.

\section{Physical Activity and Student Perception of Their Fitness Feedback}

In students with significantly diminishing intrinsic motivation toward physical activity at Time 3, it was also expected to see a decrease in physical activity levels. However, overall physical activity levels did not change significantly at any of the time points for any group (Figure 3).
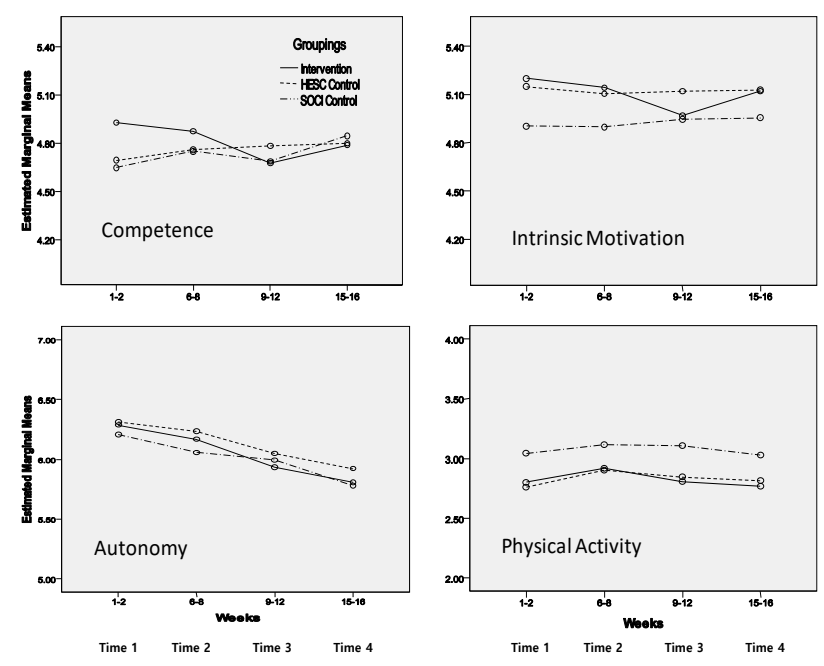

Figure 3: Self-determination theory constructs in intervention and control groups across four time points.

Examination of the perception variable showed almost one-third (32\%) of the intervention students reported a negative perception of their fitness feedback and $68 \%$ reported a positive perception. Therefore, if trying to explain the significant drop in intrinsic motivation by simply a larger number of students who viewed their feedback as negative, this would not be a valid argument as more students actually perceived their fitness assessment feedback as positive.

Among those who perceived their feedback negatively, intrinsic motivation $(t(59)=4.01$, $p<.001)$, competence $(t(59)=3.89, p<.001)$, and physical activity $(t(59)=2.95, p=.005)$ decreased significantly between participating in the fitness assessment, Time 2 and when they received their 
fitness assessment feedback, Time 3 (Figure 4). No significant changes in intrinsic motivation, competence, autonomy and physical activity were seen among those who perceived their fitness assessment feedback positively.
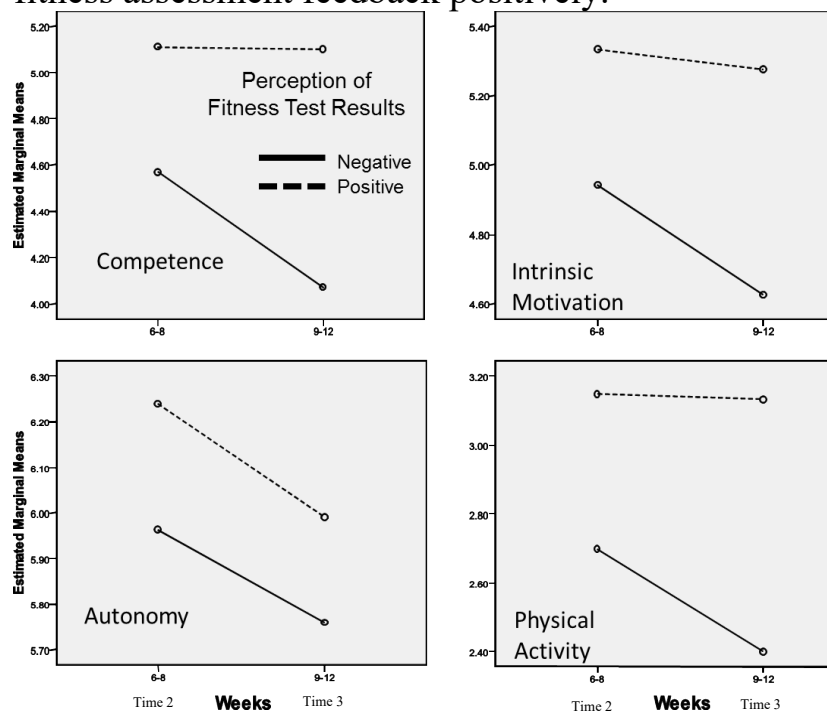

Figure 4: The negative and positive perception groups within the intervention group at Time 2 (6-8 weeks) and Time 3 (9-12 weeks)

\section{Discussion}

Based on the SDT theory one would expect, as competence decreases, intrinsic motivation is diminished, thereby decreasing physical activity. What is telling from this study is that receipt and review of one's fitness assessment feedback which one perceives as negative predicted this decrease, however there was no corresponding increase in competence, intrinsic motivation or physical activity for those who perceived the feedback of their fitness assessment as positive. Furthermore, these results regarding competence and intrinsic motivation indicate that even though intuitively one may believe fitness assessment feedback can be the sort of feedback that enhances one's feelings of competence and intrinsic motivation toward physical activity, perhaps it is not. Apparently, the feedback in some way made at least some students feel less competent. This is supported in the literature as Burgers and colleagues (2015) found in their study "negative feedback decreases feelings of competence" (p.98). One could speculate that just the act of performing the fitness assessment may have diminished competence; however this was controlled for by surveying the students at Time 2 (right after they took the fitness test) and no significant change from Time 1 to Time 2 was seen. The results of this study are similar to Whitehead and Corbin's (1991) findings where negative bogus feedback decreased the constructs of SDT and CET. However, our results differ from their study in that they also saw an enhancement of the constructs with positive bogus feedback whereas in this study no significant change was seen.

Perhaps this study did not see a change in physical activity as a fitness assessment did not relate to physical activity as was thought. For example, if one observed a student playing soccer and gave him feedback on his soccer skills one would expect this feedback to affect his perceived competence regarding how he plays soccer and then also affect his intrinsic motivation towards soccer and how often he engages in soccer. However, in this study we are providing feedback on fitness and then measuring competence and intrinsic motivation toward physical activity. Experts in the field note that fitness is categorized as a product and physical activity as a process (Corbin, et al., 2000). Even though fitness and physical activity are related in general, it may not be as was speculated with regard to applying the self-determination theory.

In addition, the term fitness, for many people may equate to something one is supposed to have in order to be healthy. Therefore, these individuals may see it as extrinsic motivation which is defined as a drive for external rewards such as money, grades, or such (Deci\& Ryan, 1985). It is seen as something they have to achieve rather than something they want to achieve. Thus, focusing on the feedback of a fitness assessment may diminish intrinsic motivation because it is a cue that physical activity is not to be done just for itself but to obtain an extrinsic reward-fitness. According to Buckworth, et al., (2007) even 
though extrinsic motivation can be a behavior change tool, intrinsic motivational techniques are generally preferred as "extrinsic rewards may encourage a more external locus of control and decrease autonomy and intrinsic motivation" (p.443).

As for the decrease in the autonomy construct, had only the health students indicated a decrease in autonomy one may have concluded that the course instruction or content may have made them feel pressured by the course instructor to be physically active to aid in their health. But since a drop in all three groups is seen, this line of thinking cannot be used to explain the decrease in autonomy. Possibly the decrease in autonomy could be due to a heavier student workload as the semester progressed. Further research focusing more on this construct is needed.

\section{Limitations}

The ability to generalize these findings is limited by the use of a restricted sample of college students with an average age of 20 years from a single university. Therefore, further research at other types of colleges located in other parts of the country would increase generalization. In addition, even though a control group outside of health courses was utilized to control for the possible influence of health curriculum, it would have strengthened the design to gather participants from a variety of other general education courses. This study also would have provided more insight had we incorporated the data of the students' actual fitness assessment feedback scores in the analysis.

\section{Implications for Practice, Future Research and Conclusion}

The results of this study have implications for the field of college health and health education and promotion. As mentioned previously fitness assessments are a common tool used in junior high and high schools as well as on college campuses (although in the college setting, they are often optional). What may be an influencing factor beyond just the actual performance of the fitness assessment is the delivery of the results, the feedback. Would the expected increase of competence and enhancement of intrinsic motivation be seen if the feedback were delivered by a person such as a teacher or coach rather than a feedback report simply passed back to students? Reflecting back to the work by Weirsma and colleagues (2008), we see the role delivery of feedback can play. Or perhaps the formatting or presentation of the feedback report itself is somehow conducive to the decrease of these competence and intrinsic motivation. Based on this study and the others highlighted, health educators and health promotion professionals in the field would benefit from consideration of how fitness assessment feedback is delivered as well as how it is perceived by the student and if any follow up might be beneficial to the student in order to positively impact their health behaviors. Further research needs to focus not only on the delivery method of feedback but also on examining groups based on the perception of their feedback. Perhaps for those that considered their feedback negative, an additional health education intervention addressing ways to increase competence toward physical activity could be explored. In fact, in their recommendations for physical education teachers conducting fitness assessments, Phillips and colleagues (2017) suggest providing students specific activities and coaching that can help improve fitness after providing feedback.

Another area of related research is on the use of health risk appraisals. This is a logical next step in this research area as these assessment tools are used in numerous health promotion settings much like fitness assessments. With that said, most of the existing research on health risk appraisals focus more on the efficacy of the tool for behavior change rather than the effect the tool has regarding motivation for the activity or for a change of behavior.

More research is needed but on the basis of this study, which so far is the only one found that 
evaluated the effects of fitness testing on intrinsic motivation, the use of fitness assessments in an attempt to increase intrinsic motivation for physical activity may need to be reconsidered.

\section{References}

American College Health Association. National College Health Assessment: Reference Group Data Report, Spring 2019. Baltimore, MD: American College Health Association: 2019. Website, https://www.acha.org/NCHA/ACHA-

NCHA_Data/Publications_and_Reports/NCHA/Data/Reports_ACHA-NCHAIIc.aspx

Brownson, R.C., Boehmer, T. K., \& Luke, D. A. (2005). Declining rates of physical activity in the United States: What are the contributors? Annual Review of Public Health, 26, 421-443. doi: 10.1146/annurev.publhealth.26.021304.144437.

Buckworth, J., Lee, E.R., Regan, G., Schneider, K.L., \& DiClemente, C.C. (2007). Decomposing intrinsic and extrinsic motivation for exercise: Application to stages of motivational readiness. Psychology of Sport and Exercise, 8, 441-461. doi: 10.1016/i.psychsport.2006.06.007

Burgers, C., Eden, A., Van Engelenburg, M.D., \& Buningh, S. (2015). How feedback boostsmotivation and play in a brain-training game, Computers in Human Behavior, 48, 94-103. ISSN 0747-5632. doi: 10.1016/j.chb.2015.01.038.

Calfas, K.J., Sallis, J.F., Lovato, C.Y., \& Campbell, J. (1994). Physical activity and its determinants before and after college graduation. Medical Exercise Nutrition Health, 3, 323-334. website, https://drjimsallis.org/measure grad.html

Carlsona, A.S., Fultona, E.J., Pratta, M., Yangb, Z., \& Adamsb, E.K. (2015). Inadequate physical activity and health care expenditures in the United States. Progress in Cardiovascular Diseases, 57, $315-$ 323. doi: 10.1016/j.pcad.2014.08.002.

California Department of Education. Overview of the California Physical Fitness Test (PFT). 2009. Retrieved from http://www.cde.ca.gov.

Centers for Disease Control and Prevention. Youth Risk Behavior Surveillance, United States, 2011. Morbidity Mortality Weekly Report, 2012;61 (No. SS-4):1-28. website, https://www.cdc.gov/mmwr/pdf/ss/ss6104.pdf

Centers for Disease Control and Prevention. National College Health Risk Behavior Survey, United States, Morbidity Mortality Weekly Report, Surveillance Summaries, 1997; 46(SS-6);1-54. website, https://www.cdc.gov/mmwr/preview/mmwrhtml/00049859.htm

Centers for Disease Control and Prevention, Division of Nutrition, Physical Activity and Obesity. Obesity Trends among U.S. Adults between 1985-2010. Available at http://www.cdc.gov/nccdphp/DNPAO/aboutus/manuscripts/index.html.

Corbin, C.B., Pangrazi, R.P. \& Frank B.D. (2000) Definitions: Health, fitness, and physical activity. The President's Council on Physical Fitness and Sports. Dept of Health and Human Services. http://www.fitness.gov/publications/digests/digest mar2000.html

Deci, E.L. (1975). Intrinsic motivation. New York: Plenum. website, https://link.springer.com/content/pdf/bfm\%3A978-1-4613-4446-9\%2F1.pdf

Deci, E.L., \& Ryan, R.M. (1985) Intrinsic mmotivation and self-determination in human behavior. New York: Plenum. website, https://www.worldcat.org/title/intrinsic-motivation-and-selfdetermination-in-human-behavior/oclc/12235854

Deci, E.L., \& Ryan, R.M. (2000). The "what" and "why" of goal pursuits: Human need and selfdetermination of behavior. Psychological Inquiry, 11, 227-268. doi: 10.1207/S15327965PLI1104_01 
Frederick-Recascino, C.M. (2002). Handbook of self-determination research. New York: Rochester Press. website, https://www.worldcat.org/title/handbook-of-self-determinationresearch/oclc/48965032.

Intrinsic Motivation Inventory (n.d.). Retrieved September 5, 2019 from Self Determination Theory website, http://selfdeterminationtheory.org

McAuley, E., Duncan, T., \& Tammen, V.V. (1989). Psychometric properties of the Intrinsic Motivation Inventory in a competitive sport setting: A confirmatory factor analysis. Research Quarterly for Exercise and Sport, 60, 48-58. doi: 10.1080/02701367.1989.10607413.

Phillips, S.R., Marttinen, R., \& Mercier, K. (2017). Fitness aassessment: Recommendations for an enjoyable student experience Strategies: A Journal for Physical and Sport Educators 30, 19-24. doi: $10.1080 / 08924562.2017 .1344168$

Ryan, R.M, \& Deci, E.L. (2000). Self-determination theory and the facilitation of intrinsic motivation, social development, and well-being. American Psychologist, 55, 68-78. doi: 10.1037/0003066X.55.1.68

Tsigilis, N., \& Theodosiou, A. (2003). Temporal stability of the Intrinsic Motivation Inventory. Perceptual and Motor Skills, 97, 271-280. doi: 10.2466/pms.2003.97.1.271.

Trends in the Prevalence of Physical Activity and Sedentary Behaviors National YRBS: 1991-2017. (n.d.). Retrieved from CDC website, http://cdc.gov/.../trends/2017 physical trend yrbs.pdf

U.S. Department of Health and Human Services. (2020), Heathy People 2030. Washington DC: U.S Department of Health and Human Services https://www.healthypeople.gov/2030

U.S. Department of Health and Human Services. Physical Activity Guidelines for Americans, 2nd edition. Washington, DC: U.S. Department of Health and Human Services; 2018. Website, https://health.gov/sites/default/files/2019-09/Physical_Activity Guidelines_2nd edition.pdf

U.S. Department of Education. Shape of the Nation. 2009. Retrieved from http://www.fitness.gov

University of Rochester, New York, Department of Psychology, Self-Determination Theory, An Approach to Human Motivation and Personality. (n.d.). Retrieved from http://www.psych.rochester.edu/SDT/index.php

Weirsma, D.L., \& Sherman, P.C. (2008). The responsible use of youth fitness testing to enhance student motivation, enjoyment and performance. Measurement in Physical Education and Exercise Science, 12, 167-183. doi: 10.1080/10913670802216148

Whitehead, J.R., \& Corbin, C.B. (1991). Youth fitness testing: The effect of percentile-based evaluative feedback on intrinsic motivation. Research Quarterly for Exercise and Sport, 62, 225-231. doi: 10.1080/02701367.1991.10608714.

Wilder, R.P., Greene, J.A., Winters, K.L., Long, W.B., Gubler, K., \& Edlich, R.F. (2006) Physical fitness assessment: An update. Journal of Long-Term Effects of Medical Implants, 16(2):193-204. doi: 10.1615/jlongtermeffmedimplants.v16.i2.90.

Corresponding Author Information

Laura Chandler -

8701 E. Foxhollow Dr.

Anaheim, CA 92808

(714) 743-7251

Lchandler@fullerton.edu 